\title{
UV CENTRAL SPIKES IN EARLY TYPE GALAXIES
}

\author{
F. BERTOLA AND M. CAPPELLARI \\ Dipartimento di Astronomia, Padova, Italy \\ D. BURSTEIN \\ Arizona State University, Tempe, USA \\ L. GREGGIO AND A. RENZINI \\ Dipartimento di Astronomia, Bologna, Italy \\ AND \\ S. DI SEREGO ALIGHIERI \\ Osservatorio Astrofisico, Arcetri, Italy
}

HST images of the E galaxies NGC 1399 and NGC 4552 and of the Sa NGC 2681 were obtained with FOC f/96 through the filters F175W, F220W, F275W and F342W. The most important discovery of these observations is the clear detection of central spikes in NGC 2681 and NGC 4552, that are prominent in each of the FOC images. No spike is detected in NGC 1399. The spikes appear unresolved by the $0^{\prime \prime} .07$ core of the Pre-COSTAR PSF, corresponding to a physical size $<\sim 2-3$ pc. We estimate $V=16.4$ for the spike in NGC 2681 and $V=18.4$ for that in NGC 4552 , or $\sim 10^{7} L_{\odot}$ in both cases. The spikes contribute less than a few percent of the UV flux sampled by IUE. We can offer three options for their nature: a central burst of star formation; effects of stellar interaction in a very dense environment; and a low level AGN. The first two options are currently entertained for the origin of the very dense star cluster at the center of our own Galaxy, whose size and luminosity are comparable to the corresponding limits for the two UV spikes (Genzel et al. 1994). A much fainter central spike $\left(\sim 10^{5} L_{\odot}\right)$ is also seen at the center of NGC 205, and appears to be consistent with a modest burst of star formation (Bertola et al. 1995).

\section{References}

Bertola, F., Bressan, A., Burstein, D., Buson, L.M., Chiosi, C., \& di Serego Alighieri, S., 1995, ApJ, in press

Genzel, R., Hollenbach, D., \& Townes, C.H. 1994, Rep. Prog. Phys. 57, 417 